\title{
Mobile-learning como estrategia de refuerzo académico en el proceso de enseñanza aprendizaje de la matemática.
}

\section{Mobile-learning as a strategy for academic reinforcement in the teaching- learning process of mathematics.}

Jorge Enrique Ortiz Acuña. ${ }^{1}$ \& Galo López Sevilla. ${ }^{2}$

\begin{abstract}
.
Introduction. Considering that low school performance in the area of mathematics affects and worries the Ecuadorian educational system, the implementation of virtual strategies that contribute to the performance of students, in the cognitive process, autonomy and experience is essential. Objective. Determine the incidence of Mobile - Learning as a strategy for academic reinforcement in the teaching-learning process of mathematics. Methodology. It corresponds to the non-experimental quantitative modality with a longitudinal design and with a correlational explanatory descriptive scope, the deductiveinductive method is attributed; In addition, the survey technique is validated (Maiza, 2018), for this purpose the SPSS Statistical Program is applied, with the Likert Scale: totally disagree (1), disagree (2), neither agree nor disagree (3), agree (4), totally agree (5), with a reliability of 0.953 . The sample under study includes 60 students from 10 to 12 years of age, who study sublevel 3: 5th and 6th grades of basic general education. Results. $63 \%$ of teachers do not strengthen the interaction between technology, motivation and knowledge, $48 \%$ do not use ICT, 50\% do not corroborate in the understanding of conceptualizations, $68 \%$ do not use the computer, do not access the

\footnotetext{
${ }^{1}$ Pontificia Universidad Católica del Ecuador | PUCE, Maestría Innovación en Educación, Tungurahua, Ambato. Ecuador, https://orcid.org/0000-0002-4279-0747, enrique.ortiz@educacion.gob.ec

2 Pontificia Universidad Católica del Ecuador | PUCE, Maestría Innovación en Educación, Tungurahua, Ambato. Ecuador, ORCID: https://orcid.org/0000-0003-4699-4875 glopez@ pucesa.edu.ec
} 
social networks and the Internet. Once the potential of Mobile - Learning is known. Conclution. The analysis shows that there is an increase from $35 \%$ to $100 \%$ that the use of active strategies will improve the development of logical and mathematical thinking.

Keywords: strategy, Mobile - Learning, teaching-learning process, academic reinforcement, mathematics, logical reasoning, problem solving.

\section{Resume}

Introducción. Considerando que el bajo rendimiento escolar en el área de la matemática afecta y preocupa al sistema educativo ecuatoriano, es trascendental la implementación de estrategias virtuales que aporten en el desempeño de los educandos, en el proceso cognitivo, la autonomía y experiencia. Objetivo. Determinar la incidencia del Mobile Learning como estrategia de refuerzo académico en el proceso de enseñanza aprendizaje de la matemática. Metodología. Corresponde a la modalidad cuantitativa de tipo no experimental con un diseño longitudinal y con un alcance descriptivo explicativo correlacional, se atribuye el método deductivo - inductivo; además, se valida la técnica de la encuesta, (Maiza, 2018), para el efecto se aplica el Programa Estadístico SPSS, con la Escala de Likert: totalmente en desacuerdo (1), en desacuerdo (2), ni de acuerdo ni en desacuerdo (3), de acuerdo (4), totalmente de acuerdo (5), con una fiabilidad del 0,953. La muestra objeto de estudio incluye a 60 estudiantes de 10 a 12 años de edad, que cursan el subnivel 3: $5^{\circ}$ y $6^{\circ}$ grados de educación general básica. Resultados. El $63 \%$ de maestros no fortalece la interacción entre la tecnología, la motivación y el conocimiento, el $48 \%$ no usa las TIC, el 50\% no corrobora en la comprensión de conceptualizaciones, el 68\% no maneja el computador, no accede a las redes sociales e Internet. Una vez conocido el potencial del Mobile - Learning. Conclusión. En el análisis se evidencia que existe un aumento desde el $35 \%$ al 100\% que el uso de estrategias activas mejoran el desarrollo del pensamiento lógico y matemático.

Palabras claves: Estrategia, Mobile - Learning, Proceso cognitivo, Proceso de enseñanza, Rendimiento académico, Razonamiento.

\section{Introducción.}

Anterior a la revolución digital, la enseñanza se cimentaba en la utilización del lápiz, el papel y libros con exuberante contenido, incluían únicamente aspectos teóricos; según Bravo (2016) en el siglo XXI, con la influencia de las tecnologías de la información y comunicación en los ámbitos de la vida cotidiana, la educación sobrelleva una progresiva transformación en un proceso que abandona el uso de papeles (hojas, folletos, textos, periódicos e impresos) y lapiceros a cambio de la pantalla táctil, el stylus y el teclado; es decir los estáticos métodos tradicionales dan paso a la motivación, el dinamismo, la creatividad, autonomía e independencia en el aprendizaje, propone posibilidades en el progreso educativo, en un enfoque global que abarca la calidad en la formación y la 
práctica de valores, componentes que aportan en la superación de obstáculos en igualdad de oportunidades.

En Ecuador, de acuerdo con el informe emitido por el Instituto Nacional de Evaluación Educativa - Ineval (2018), en las puntuaciones de PISA, que evalúa a cada estudiante, expone en el resultado que en un alto porcentaje se encuentran por debajo del nivel básico de competencia en lectura (51\%), ciencias (57) y matemáticas una cifra alarmante del $71 \%$; se deduce que existen barreras que afectan en el pensamiento matemático avanzado, el entendimiento, la comprensión, el dominio de las operaciones simbólicas y formales. Según (Ramírez, 2016) menciona como factores influyentes en el refuerzo académico, el salón de clase no atractivo en el área visual y físico (13,86\%); inadecuados hábitos de estudio (13.52\%), escasa optimización del tiempo en el análisis de contenidos en el hogar (12.5\%); complicaciones sentimentales (3.28\%), carencia de apoyo familiar (2.56\%); por lo tanto, la enseñanza mecánica y memorística, no ayuda en el desarrollo de las capacidades cognitivas de los educandos, afectando en el desempeño educativo.

De acuerdo con Rodríguez \& Rey (2017) entre las causas influyentes en el bajo rendimiento escolar en el área de matemática sobresalen las siguientes:

- Docentes que utilizan métodos didácticos tradicionalistas.

- Maestros que no utilizan métodos activos de enseñanza.

- Docentes renuentes al uso de la tecnología.

- Desinterés en temas de auto capacitación.

- Estudiantes con problemas de aprendizaje en el área de matemática.

- Educandos desmotivados y/o desinteresados por su formación académica.

- Estudiantes con deficiencia en razonamiento lógico matemático.

- Estudiantes con dificultades para resolver las operaciones matemáticas elementales.

- Bajo nivel cultural de los padres de familia.

- Desinterés de padres y representantes por el rendimiento escolar de sus representados.

La educación, al centrase en el desarrollo del ser humano, tiene la finalidad de alcanzar la formación académica con visión científica y humanista a través de la investigación, la tecnología, la innovación y difusión de los saberes; para Tamayo (2020) el uso de dispositivos móviles permite introducir en las instituciones el Mobile learning que favorece en el saber en los diferentes ámbitos y niveles, coadyuvando en la transformación digital, al contribuir en la aplicación de métodos y metodologías que impactan en la calidad educativa; por lo expuesto en el presente estudio se plantea el objetivo, determinar la incidencia del mobile - learning como estrategia de refuerzo académico en el proceso de enseñanza y aprendizaje de la matemática en estudiantes de básica media de la Escuela General Córdova. 


\section{Profundización en el concimiento.}

\section{Tecnología de la información y comunicación, herramientas digitales que benefician en los conocimientos de los educandos.}

Las tecnologías de la información y comunicación con el pasar del tiempo se han constituido en un pilar esencial de la sociedad del conocimiento y la educación; el criterio de Saquinga (2019) incluye, es un medio que favorece en el intercambio de saberes y experiencias, considerando que en el sistema educativo actual presenta nuevos desafíos, que requieren el empleo de metodologías innovadoras que apoyadas en progresos tecnológicos favorecen en las competencias del estudiante, de esta forma mejora su comprensión, facilita la resolución de ejercicios y desarrolla el pensamiento con una visión integradora; en líneas generales, se deduce que los entornos digitales han mejorado las habilidades en el acceso, la evaluación y organización de contenidos, al proporcionar medios enriquecedores, que admiten a los estudiantes participar, informar (comunicar), intercambiar, dialogar presentar ideas; mientras el docente asume el liderazgo pedagógico, didáctico y metodológico en la continuidad de la formación.

De acuerdo con Guiza (2011), Aguirre \& Ruiz (2012) y Arcavi (2018) las herramientas digitales apoyan en la enseñanza al incluir "recursos, internet, blogs, wikis, webquest, foros, chat, mensajes, videoconferencia y varios canales de comunicación que benefician en la gestión de la información. Por otro lado, la tecnología educativa a través de entornos virtuales fortalece en la creatividad e innovación, y principalmente el acceso a programas educativos con prioridad en la identificación y utilización de materiales, recursos e instrumentos electrónicos para el desarrollo de capacidades y habilidades básicas mediante la ejercitación, práctica y razonamiento que conlleva a las solución de problemas complejos en ambientes telemáticos.

Las herramientas tecnológicas benefician en la alfabetización digital de los educandos, maestros y familias, su uso didáctico (software) aporta en la comunicación, ejercitación y orientación del aprendizaje, beneficiando en los procesos cognitivos, para conseguir mejores aprendizajes, al activar los conocimientos previos (Zamora, Soares, Echeverria, Hernández, \& Mijangos, 2015). En general, por su valor pedagógico y metodológico se emplea en la evaluación, motivando al estudiante en el manejo de imágenes, vídeos y representaciones, elementos que permiten alcanzar un alto potencial cognitivo.

Para los investigadores López (2018); Ruangvanich \& Nilsook (2018) los recursos tecnológicos y las aulas virtuales benefician en el nivel intuitivo, el desarrollo de habilidades comunicativas, despiertan el interés y participación en clases, mejora la autoestima y motivación por aprender, proporciona mayor efectividad a la educación escolarizada e intensifica la oferta educativa; del mismo modo Forero (2018) propone que la generación de materiales educativos innovadores conllevan al razonamiento conceptual mediante la observación de prácticas reales encaminadas al conocimiento significativo. No obstante, Blanco, Nieto, \& Caballero (2015) afirman, la revolución tecnológica en el entorno educativo agiliza el trabajo docente y el aprendizaje de los estudiantes. En esta 
perspectiva, la integración de herramientas digitales disminuye el abandono escolar, refuerza el rendimiento en las diferentes asignaturas y eleva la calidad en la formación.

Las tecnologías en el área educativa mediante diversas aplicaciones facilitan la enseñanza y el aprendizaje de las matemáticas, según Boaler (2019) y Mojarro (2019) el sujeto aprende en el manejo de sistemas de representación que apoyan en el proceso de comprensión; desde un aspecto dinámico promueve la manipulacion de los objetos matemáticos y sus relaciones, así el aprendiz construye experiencias propias. De acuerdo con el criterio de los autores mencionados, el avance de las nuevas funcionalidades integradas en dispositivos electrónicos es un factor que trasciende en el ámbito educativo al englobar diferentes estrategias de Mobile Learning, herramienta que apoya en la independencia tecnológica de los contenidos.

\section{Mobile-learning como estrategia de refuerzo académico en el proceso de enseñanza aprendizaje de la matemática.}

Como afirma Hinojo, Aznar, Cáceres, \& Romero (2019), "la literatura existente en referencia a Mobile Learning ha evolucionado de forma paulatina desde una visión tecnocéntrica a la percepción formativa; los investigadores Yang \& Huang (2016) manifiestan, "es cualquier acción educativa, finalmente, Chávez \& Chévez (2019) en la perspectiva pedagógica puntualizan "las tecnologías son difundidas junto con las redes que facilitan, apoyan y mejoran la enseñanza". En definitiva las estrategias de Mobile Learning activa los ambientes de aprendizaje, abordan el trabajo colaborativo, la comunicación sincrónica y asincrónica, que potencia el proceso de gestión basado en competencias.

Según, Panaqué \& Guerrero (2017) las tecnologías móviles en cualquier sitio, refuerza la creatividad propia; Basantes, Gallegos, \& Benítez, (2017) "proporciona herramientas, recursos, medios y formatos"; estrategias didácticas que viabilizan en la cimentación del conocimiento: "aulas virtuales, blogs didácticos, evaluaciones online, realidad virtual, entornos 3d". Por tanto el éxito en la educación deriva de la habilidad en la integración de la tecnología al plan de estudios y la generación de experiencias individuales, mediante la transformación del aula en un ambiente colaborativo a través del diseño de ambientes educativos digitales centrados en el estudiante que es protagonista de su formación.

Según Fuenmayor \& Villasmil (2008), Paredes (2017) y Batista (2015), el Mobile learning es un modelo pedagógico empleado en el aprendizaje de diversas asignaturas, sobretodo en el área matemática, que tiene por propósito orientar las prácticas educativas mediante dispositivos móviles; las actividades propuestas motivan en el progreso de las funciones básicas: atención (percepción), concentración, memoria (corto, largo y mediano plazo), pensamiento (abstracto, creativo, argumentativo) y razonamiento (lógico), lenguaje. Sobre la base de las ideas expuestas, beneficia en los procesos de asimilación, incrementa la comprensión de conceptos y procedimientos matemáticos, que facilita la habilidad para resolver problemas de menor y mayor complejidad. 
Afirman Reina \& La Serna (2020), Trabaldo (2015) el Mobile Larning es una metodología utilizada en dispositivos móviles: teléfonos, tabletas, PocketPC, iPod, requiere de una conectividad inalámbrica, "WiFi" de alta capacidad de almacenamiento; el progreso de la tecnología móvil ha concebido una mejora en la educación para respaldar a los educadores y optimizar sus prácticas de instrucción dentro y fuera del salón de clases sin importar las restricciones del tiempo y espacio, además, perfecciona el trabajo educativo pedagógico, que conlleva a un aprendizaje autónomo en relación con el avance de las nuevas tecnologías de la información y la comunicación

Según O’Malley (2016) el Mobile-Learning como estrategia de refuerzo académico establece la sincronización con varios dispositivos, en el aprendizaje de las matemáticas favorece en el acceso a los contenidos académicos, potencia la motivación por aprender desde el celular, medio electrónico que permite la utilización de diversas Apps; en los escenarios pedagógicos brinda nuevas disyuntivas de interacción, que permiten alcanzar la flexibilidad e independencia en la modificación de hábitos de estudio que renuevan la eficacia en el rendimiento.

\section{Metodología.}

La metodología corresponde a la modalidad cuantitativa de tipo no experimental con un diseño longitudinal y con un alcance descriptivo, explicativo y correlacional. Se utilizó el método deductivo - inductivo utilizando encuestas validadas estadísticamente con pruebas de fiabilidad que permitieron indagar sobre la temática propuesta y analizar el uso del Mobile-Learning para alcanzar aprendizajes significativos, el aporte se enmarcó en educación y tecnología.

Desde el enfoque cualitativo profundiza en la comprensión de los significados y definiciones relacionadas con la situación que presentan las personas, además permite analizar la realidad y las características propias del entorno educativo, mediante las inferencias y valoración entorno a los resultados de la indagación; según López (2018), es cuantitativo porque admite la recolección y el análisis de datos para contestar una o varias preguntas establecidas en el estudio.

De igual forma se desarrolló la recolección de información mediante instrumentos estructurados y validados, aspecto que benefició en el análisis estadístico y porcentual, empleando para el efecto el tipo de investigación de campo consolidada en la Escuela General Córdova.

\section{Participantes.}

La muestra objeto de estudio incluye a 60 educandos entre los 10 a 12 años de edad años de edad, que cursan el subnivel 3: $5^{\circ}$ y $6^{\circ}$ grados de educación general básica de la Escuela General Córdova. 


\section{Instrumentos.}

En la recolección de la información relacionada con el Mobile-Learning como estrategia de refuerzo académico en el proceso de enseñanza aprendizaje de la matemática se aplicó la técnica de la encuesta, mediante la adaptación del cuestionario (tabla 1) que incluye 18 interrogantes propuestas por (Maiza, 2018).

Los nueve primeros ítems corresponden a la variable independiente: El Mobile learning como estrategia de refuerzo académico que según Fuenmayor \& Villasmil (2008), Paredes (2017) y Batista (2015), es un modelo pedagógico empleado en el aprendizaje de diversas asignaturas; sobre todo en el área matemática tiene por propósito orientar las prácticas educativas mediante dispositivos móviles, que integran los recursos tecnológicos e internet, motivando al estudiante en el progreso de las funciones básicas: atención (percepción), concentración, memoria (corto, largo y mediano plazo), pensamiento (abstracto, creativo, argumentativo) y razonamiento.

Las categorías hacen referencia a las prácticas educativas (recursos tecnológicos, interacción con el conocimiento, refuerzo académico); motivación (comprensión, teléfonos móviles y Smartphone, aparatos reproductores y videojuegos); tecnología (dispositivos móviles, computador e internet)

Los otros nueve ítems integran la variable dependiente: el proceso de enseñanza de la matemática, en su conceptualización los autores (Aznar \& García, 2018), (Vidal, 2018) manifiestan que desde los escenarios pedagógicos el uso de tecnología móvil e inalámbrica, brinda nuevas disyuntivas de interacción, logrando la flexibilidad e independencia, a través de la modificación de hábitos de estudio, la incorporación de métodos y herramientas que mejoran la eficacia en el rendimiento.

Las categorías abarcan los escenarios pedagógicos (solución de ejercicios, uso de herramientas tecnológicas y entendimiento) interacción (refuerzo de contenido, razonamiento y compartir); rendimiento (participación, trabajos en equipo y estrategias activas).

Tabla 1. Ítems aplicados a los estudiantes

\begin{tabular}{ll}
\hline \multicolumn{1}{c}{$\mathbf{N}^{\mathbf{0}}$} & \multicolumn{1}{c}{ Ítems } \\
\hline 1 & ¿La institución educativa cuenta con recursos tecnológicos para impartir refuerzo pedagógico? \\
\hline 2 & $\begin{array}{l}\text { ¿Considera usted que los recursos tecnológicos permiten una mayor interacción con el } \\
\text { conocimiento, motivando en el aprendizaje? }\end{array}$ \\
\hline 3 & $\begin{array}{l}\text { ¿El maestro, utiliza las TIC, como recurso del refuerzo académico de la Matemática con los } \\
\text { estudiantes? }\end{array}$ \\
\hline 4 & $\begin{array}{l}\text { ¿La utilización de herramientas tecnológicas le ayuda a comprender mejor los temas } \\
\text { matemáticos vistos en el aula? }\end{array}$ \\
\hline 6 & $\begin{array}{l}\text { ¿El docente utiliza dispositivos móviles (teléfonos móviles y Smartphone) para impartir el } \\
\text { refuerzo académico de Matemática? }\end{array}$ \\
\hline ¿La utilización de nuevas tecnologías (teléfonos móviles, aparatos reproductores y video \\
juegos) benefician en el refuerzo de contenidos matemáticos?
\end{tabular}


Tabla 2. Ítems aplicados a los estudiantes (continuación)

\begin{tabular}{|c|c|}
\hline $\mathbf{N}^{0}$. & Ítems \\
\hline 7 & $\begin{array}{l}\text { ¿La utilización de dispositivos móviles beneficia en los refuerzos académicos que usted recibe } \\
\text { en el área de matemática? }\end{array}$ \\
\hline 8 & ¿En el proceso de enseñanza el docente de matemática se utiliza el computador? \\
\hline 9 & ¿Tiene usted computador en la casa con acceso al internet? \\
\hline 10 & ¿Usted emplea la Tablet para aprender matemáticas en el aula de clase? \\
\hline 11 & $\begin{array}{l}\text { ¿Mediante las redes sociales (WhatsApp y Facebook) pregunta a sus compañeros las tareas o } \\
\text { solución de ejercicios matemáticos? }\end{array}$ \\
\hline 12 & ¿Utiliza el internet o software, para comprender de mejor la Matemática? \\
\hline 13 & ¿Usted emplea el internet para reforzar el conocimiento en los contenidos de matemática? \\
\hline 14 & $\begin{array}{l}\text { ¿El docente realiza ejercicios de razonamiento matemático mediante dispositivos móviles en } \\
\text { el aula de clase? }\end{array}$ \\
\hline 15 & ¿Considera que el docente crea espacios virtuales para compartir aprendizajes? \\
\hline 16 & ¿La aplicación de la tecnología promueve su participación en la construcción del aprendizaje? \\
\hline 17 & ¿En la enseñanza de matemática el maestro le permite realizar trabajos en equipo? \\
\hline 18 & $\begin{array}{l}\text { ¿Cree usted que las clases de matemáticas serían más atractivas si se las combina con } \\
\text { aplicaciones móviles que apoyen en su aprendizaje? }\end{array}$ \\
\hline
\end{tabular}

Compilado por: investigador, tomado de (Tapia, 2018)

El instrumento en el presente estudio fue validado, en el programa estadístico SPSS, evidenciado en la tabla 2; se emplea la escala de Likert: totalmente en desacuerdo (1), en desacuerdo (2), ni de acuerdo ni en desacuerdo (3), de acuerdo (4), totalmente de acuerdo (5).

Tabla 3. Valores estadísticos

Estadísticos de fiabilidad

\begin{tabular}{lcrr} 
Alfa de Cronbach & \multicolumn{2}{c}{$\begin{array}{c}\text { Alfa de Cronbach basada en los } \\
\text { elementos tipificados }\end{array}$} & N de elementos \\
\hline &, 953 &, 953 & 18
\end{tabular}

Elaborado por: investigador

Con un total de 60 estudiantes y 18 elementos se obtiene la fiabilidad en un Alfa de Cronbach del 0.953, (tabla 2).Según Villanueva,(2016), el valor de fiabilidad en el estudio que incluye una investigación básica oscila entre 0.7 y 0.8 ; por tal razón equivale al criterio de excelente.

\section{Resultados.}

\section{Análisis.}

Antes y después de utilizar como herramienta para la enseñanza de matemática el Sitio Google (https://sites.google.com/view/reforzandonuestroconocimiento/inicio), con sustento en los resultados obtenidos de la encuesta aplicada a los estudiantes de quinto y sexto grado de Educación General Básica de la Escuela General Córdoba (grupo de control y experimental) sobresalen los siguientes resultados:

Según el análisis efectuado en el pretest, se obtiene que el 65\% afirma que la institución no cuenta con recursos tecnológicos para impartir refuerzo pedagógico, por tal razón los 
docentes no aplican materiales que beneficien en el rendimiento, el 52\% no logra la interacción con el conocimiento. En el post test en referencia a las prácticas educativas, motivación (teléfonos móviles y Smartphone, aparatos reproductores y video juegos) y tecnología, (dispositivos, computador e internet se determina que el 100\% de maestros trabajan con estrategias informáticas que contribuyen en la comprensión de temas matemáticos; además, utilizan herramientas como la computadora para apoyar en la toma de decisiones, el razonamiento y la solución de problemas complejos.

Ante lo expuesto (De la Cruz Ramírez, Duran, Romero, \& Álvarez, 2020) menciona que la insuficiente creación de escenarios virtuales afecta en la aprehensión e interiorización de definiciones tratados en el contexto escolar, lo que genera dificultades en la descomposición, codificación, sistematización y secuenciación de procedimientos. Es decir, los ambientes de trabajo online despiertan el interés del educando en el acceso a aulas o campus informáticos.

Los resultados en el pretest indicaron que el $66 \%$ de estudiantes mantiene dificultades en la comprensión de los temas matemáticos vistos en el aula; en referencia a la utilización dispositivos (teléfonos móviles y Smartphone) el 35\% expone que los maestros no aplican estrategias tecnológicas para impartir el refuerzo académico de Matemática, un porcentaje del $65 \%$ no utiliza el internet, acción que repercute en el desempeño escolar, las habilidades cognitivas, la reflexión, el razonamiento y la toma de decisiones; en el post test en cuanto escenarios pedagógicos para la solución de ejercicios el 100\% aplica herramientas virtuales que refuerzan el entendimiento de contenidos, la participación y el trabajo en equipo; según (Angulo, Arteaga, \& Carmenates, 2020) el dominio de definiciones y conceptualizaciones matemáticas son parte esencial en la formación del educando; (Rodríguez \& Vaca, 2018), por esta razón, la capacitación a los docentes beneficia en la cimentación del saber, la percepción, autonomía e innovación.

Cabe resaltar que en el pretest el $46 \%$ de estudiantes mediante las redes sociales no preguntan a sus compañeros las tareas o solución de ejercicios; mientras el 35\% no emplea el internet para reforzar el conocimiento en los contenidos; el 16\% está totalmente de acuerdo que el docente no realiza prácticas de razonamiento matemático con dispositivos en el aula, acciones que generan barreras en la creación de espacios virtuales para compartir y participar en la construcción del aprendizaje.

En el post test, el 100\% utilizan material educativo en la adquisición de informaciones, experiencias el desarrollo de actitudes y adopción de normas de conducta. Con respecto a los resultados anteriores (Delgado \& Inlasaca, 2019) clarifica que las herramientas tecnológicas como el Mobile-learning son necesarias en el refuerzo académico de la matemática, por tanto, es responsabilidad de los maestros la capacitación y actualización en el contexto tecnológico para la aplicación de recursos y materiales innovadores.

\section{Correlación.}

Mediante la prueba de correlación de Spearman (rho) se determina si las dos variables están correlacionadas, es decir, si los valores de la variable independiente tienden a ser 
más altos o más bajos para valores más altos o más bajos de la variable dependiente como se muestra en los resultados en la tabla 5.

\begin{tabular}{|ll|r|r|r|r|r|r}
\multicolumn{7}{c}{ Tabla 4. Correlación Spearman } \\
Correlaciones \\
\end{tabular}

**. La correlación es significativa en el nivel 0,01 (bilateral).

Elaborado por: investigador

Se realiza una correlación entre la variable independiente y la variable dependiente considerando las 6 dimensiones basadas en el desempeño por: prácticas educativas, motivación, tecnología, escenarios pedagógicos, interacción, y rendimiento.

Al realizar la prueba estadística se observa que, si existe una correlación entre la variable independiente y la variable dependiente, las diferencias resultaron estadísticamente significativas entre las dimensiones interacción y rendimiento con un valor de correlación positiva muy fuerte (.990) y un valor de significancia de (,000), tomando en consideración que la implementación del Mobile-Learning con motivación, tecnología, produce un cambio significativo en el razonamiento lógico - matemático, y la capacidad para resolver problemas.

Además, se identifica una correlación positiva muy fuerte $(>0,90)$ y un valor de significancia asintótica bilateral $(0,00)$, donde se acepta la hipótesis de investigación (Hi) y se rechaza la hipótesis nula (Ho).

\section{Verificación de la hipótesis.}

La verificación de la hipótesis, se emplea el modelo lógico, el modelo estadístico y discusión de resultados. 
Modelo Lógico. Hipótesis Nula. $\mathrm{H}_{0}$ : el Mobile Learning como estrategia de refuerzo académico no mejora los procesos de enseñanza aprendizaje de la matemática en los estudiantes de básica media de la Escuela General Córdova.

Hipótesis alternativa. $\mathrm{H}_{1}$ : el Mobile Learning como estrategia de refuerzo académico si mejora los procesos de enseñanza aprendizaje de la matemática en los estudiantes de básica media de la Escuela General Córdova.

Tabla 5. Resumen de prueba de hipótesis

\begin{tabular}{|c|c|c|c|c|}
\hline \multicolumn{5}{|c|}{ Resumen de prueba de hipótesis } \\
\hline & Hipótesis nula & Test & Sig. & Decisión \\
\hline 1 & $\begin{array}{l}\text { La mediana de las diferencias entre } \\
\text { Mobile Learning como estrategia de } \\
\text { refuerzo académico y Procesos de } \\
\text { enseñanza aprendizaje de la matemática } \\
\text { es igual a } 0\end{array}$ & $\begin{array}{l}\text { Prueba de } \\
\text { Wilcoxon de los } \\
\text { rangos con } \\
\text { signo de } \\
\text { muestras } \\
\text { relacionadas }\end{array}$ &, 000 & $\begin{array}{r}\text { Rechazar la } \\
\text { hipótesis nula }\end{array}$ \\
\hline
\end{tabular}

Se muestran las significancias asintóticas. El nivel de significancia es , 05

Elaborado por: investigador

Con 60 casos válidos, equivalente al 100\%, en el resumen de prueba de hipótesis la significación se encuentra debajo del error 0,05. Por tanto, se rechaza la hipótesis nula. El Mobile Learning como estrategia de refuerzo académico no mejora el proceso de enseñanza aprendizaje de la matemática en los estudiantes de básica media de la Escuela General Córdova.

Se sume la hipótesis alternativa. El Mobile Learning como estrategia de refuerzo académico si mejora el proceso de enseñanza aprendizaje de la matemática en los estudiantes de básica media de la Escuela General Córdova.

El estudio se efectuó en un periodo de 4 meses (Febrero - Mayo 2021) con estudiantes de quinto y sexto grado de Educación General Básica, en el sitio Google Sites se incluyó cuatro bloques:

- Adición y sustracción

- Multiplicación

- División

- Operaciones combinadas

Las herramientas y recursos tecnológicos utilizados fueron los Videos de YouTube, herramienta que apoya en el proceso de aprendizaje de adición, sustracción, multiplicación y división, que promueve la apropiación del conocimiento, la concentración, la atención y las habilidades cognitivas. Live Worksheets, admite la digitalización de diferentes actividades (selección múltiple, grabación de mensajes, presentaciones, elección de listas, respuestas con textos o números), constituyéndose en 
un diseño interactivo que mediante dispositivos electrónicos contribuye en el razonamiento, el pensamiento y la comunicación.

El Genially, favorece en la elaboración de presentaciones interactivas, al estilo del PowerPoint. Wordwall, es utilizado para generar actividades participativas un sistema de pantallas, contiene el crucigrama, el laberinto, el avión entre otros elementos. Quizizz, herramienta de gamificación aplicada en la evaluación a los estudiantes mediante cuestionarios lúdicos y divertidos.

Otro criterio significativo para la didáctica fue el espacio virtual que favoreció en el desarrollo intelectual de los estudiantes, contribuyendo en la lógica, el razonamiento, el pensamiento y la abstracción, mediante la realización de ejercicios prácticos que contuvieron: presentaciones y definiciones concernientes a los bloques curriculares: adición y sustracción, multiplicación, división y operaciones combinadas. Para Sosa (2021) Las herramientas virtuales en las matemáticas generan actitudes que benefician en la seguridad de los procedimientos y la confianza en los resultados obtenidos.

Con el fin de explorar las actividades como estrategia de aprendizaje, las evaluaciones se realizaron mediante las herramientas digitales WEB 2.0 Live Worksheets y Quizizz, elaborados en un tiempo de dos semanas, cada sesión tiene una duración de cuarenta minutos; los temas fueron compartidos previamente por el docente a través de la proyección en la pantalla, en referencia a la asistencia los estudiantes se conectan de manera virtual en la aplicación Microsoft Teams, desde esta percepción, las matemáticas desarrollan la capacidad del pensamiento, orientan en encontrar soluciones a los problemas en situaciones complejas.

Es fundamental señalar que los estudiantes han adquirido destrezas para comprender las matemáticas, de esta forma encuentran soluciones lógicas y razonadas a situaciones de la vida cotidiana.

\section{Presentación del espacio virtual}
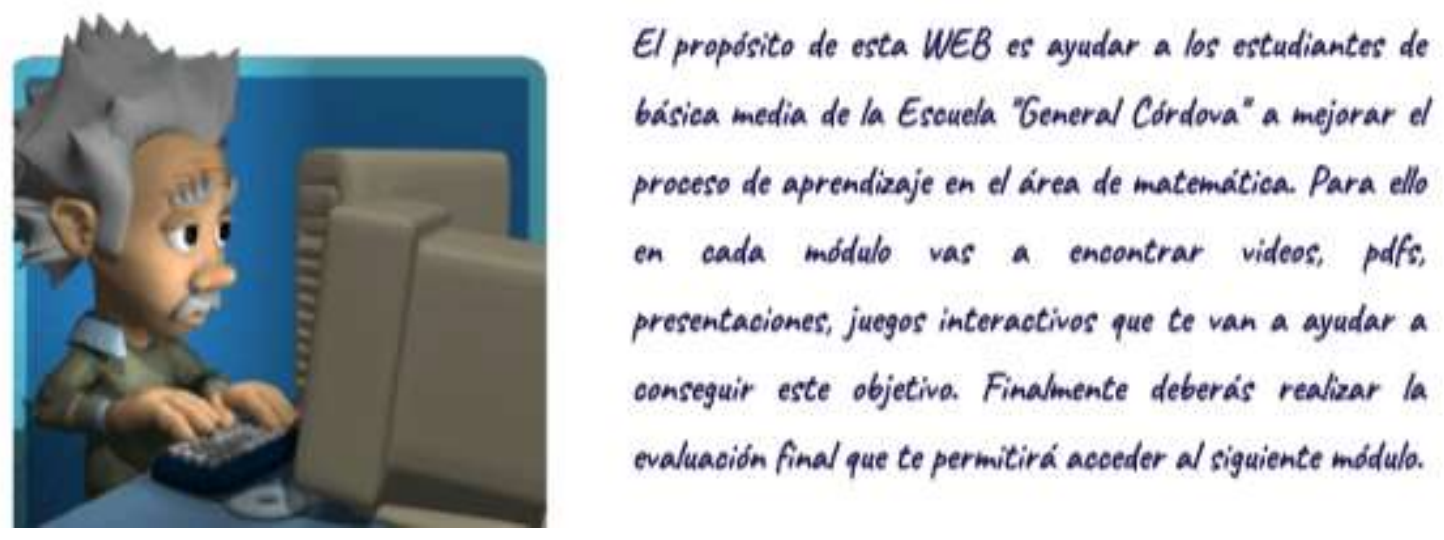

Ilustración 1. Inicio

Fuente: Escuela General Córdova 
Contenidos

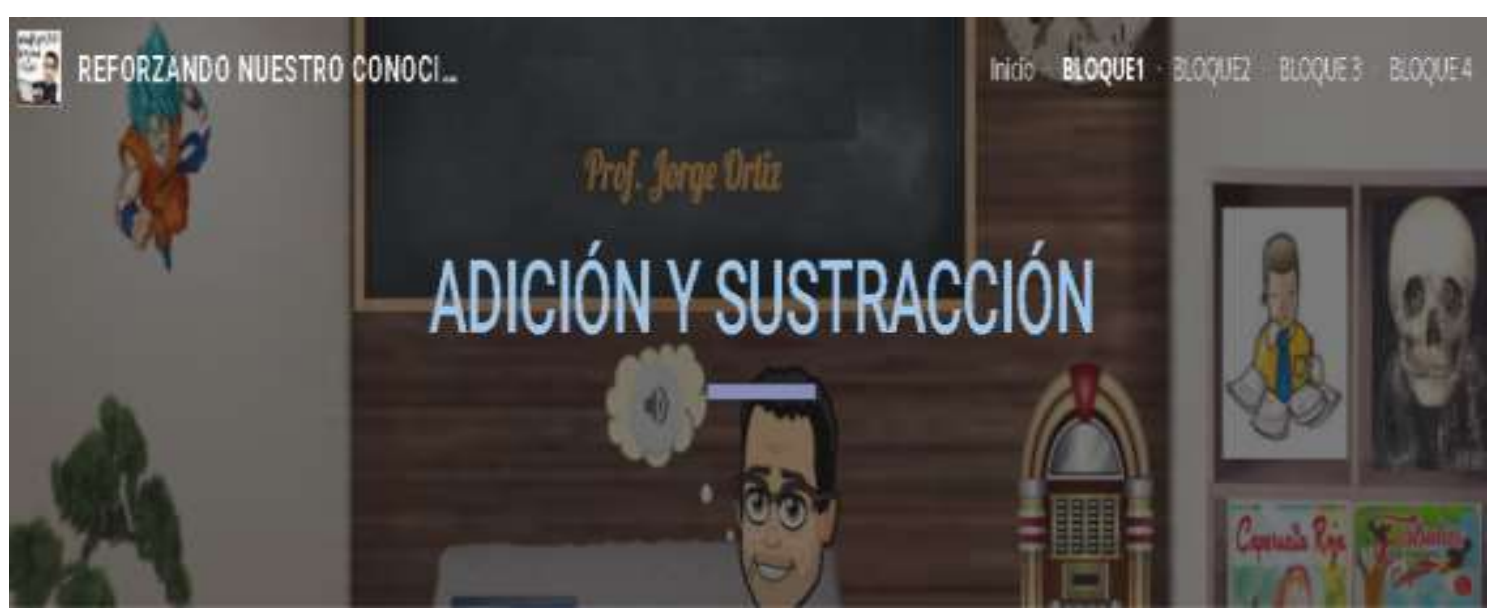

Ilustración 2. Contenidos: Adición y sustracción. Multiplicación. División. Operaciones combinadas Fuente: Escuela General Córdova

Introducción

OSUS TERMINOS:

\section{- SUMANDO \\ OSUMANDO \\ OSUMA O TOTAL}

Pagine 9 de ? $-\mathbf{a}+$

RECUERDALOS A

LA HORA DE

SUMAR.

Ilustración 3. Introducción. Definición de términos

Fuente: Escuela General Córdova

Desarrollo

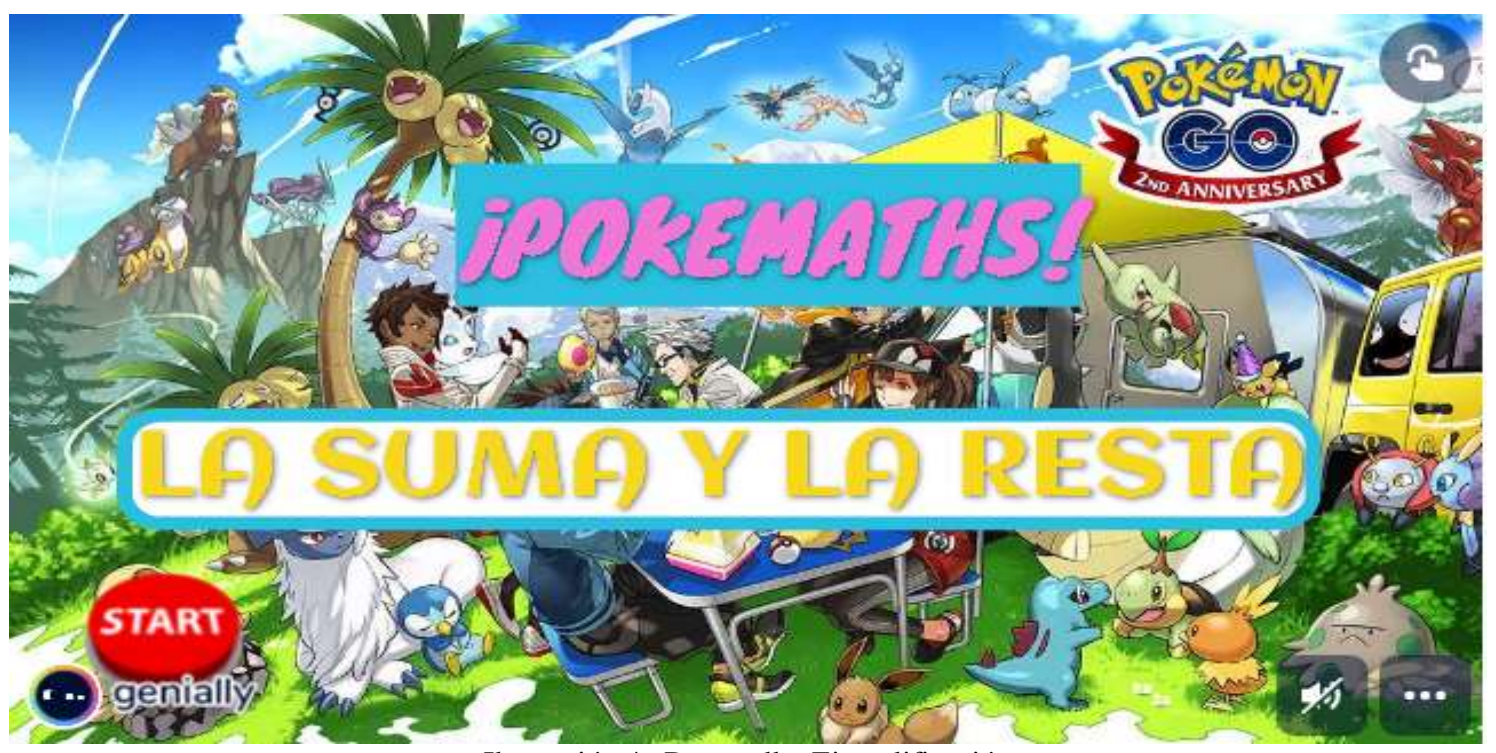

Ilustración 4. Desarrollo. Ejemplificación

Fuente: Escuela General Córdova 


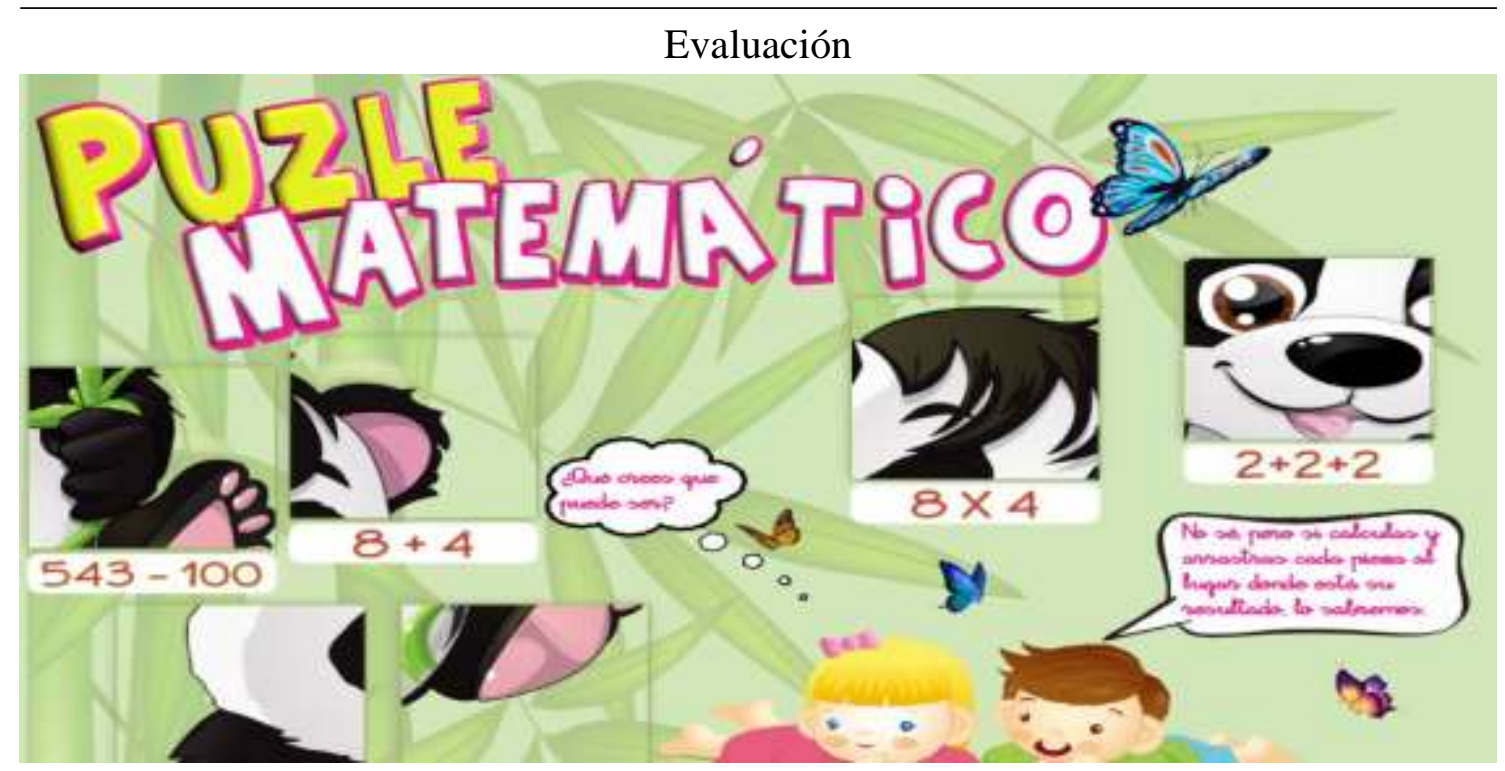

Ilustración 5. Evaluación

Fuente: Escuela General Córdova

\section{Discusión}

Es trascendental que los docentes en las instituciones educativas utilicen recursos tecnológicos para impartir refuerzo pedagógico, así, se logrará integrar los conocimientos, la teoría y la práctica en la instrucción de matemática; teniendo en cuenta el criterio descrito, Martínez (2016) manifiesta, los dispositivos móviles brindan nuevas oportunidades para crear alternativas de aprendizaje, al permitir un acceso práctico e individualizado al mundo de la información y la comunicación (pág. 17). Mientras Scopeo (2012) menciona, el Mobile-Learning, motiva en el desarrollo del pensamiento crítico y reflexivo para interpretar y solucionar problemas de la vida real; por tanto, según López (2019) el uso de tecnologías en contextos educativos apoya en el trabajo autónomo, la comprensión e internalización de los conceptos matemáticos.

En referencia a la utilización de dispositivos: teléfonos móviles, Smartphone, aparatos reproductores y video juegos benefician en el refuerzo de contenidos matemáticos, criterio que es fortalecido por Giulianelli, Vera, Rodríguez, Trigueros, González \& Pons (2012), los dispositivos ayudan a enfrentar el desafío de una sociedad que brinda nuevas posibilidades entre la diversidad de hardware y software existentes (pág. 24). Para Bravino \& Margaria (2014) en definitiva las tecnologías dan respuesta a las necesidades constantes de acceso a la información y de comunicación, forman parte del equipamiento personal de los usuarios, en su vida social, laboral y educativa (pág. 94).

Ante lo expuesto, afirman Vidal, Gavilondo, Rodríguez, \& Cuéllar (2015), el Mobile Learning configura una metodología sustentada en el uso de celulares u otros dispositivos (agendas electrónicas, tabletas o tablets) conectados a Internet. Este tipo de aprendizaje constituye una convergencia de modelos de educación a distancia y presencial, y el uso de las tecnologías inalámbricas que brindan renovadas alternativas de interacción y acceso a contenidos educativos (pág. 672). Según Gallardo, Iñiguez (2017) el uso de 
nuevos mecanismos de enseñanza que incorporan diferentes estímulos que contribuyen a lograr una mejor atención (pág. 28).

El uso de dispositivos móviles en educación es un elemento esencial en la construcción del conocimiento, con su utilización se incrementan las posibilidades de interactuar con los miembros del grupo, se optimiza la comunicación; Olmedo \& de Alzúa (2015) en este contexto de innovación y transformación tecnológica el profesorado está implementando estrategias didácticas digitales que conllevan al cambio en la escuela (pág. 88). Tamayo \& Álvarez (2017) afirman que el aprendizaje requiere de aptitudes tecnológicas por parte de los profesores, para lograr un acercamiento y adoptar nuevos conocimientos y competencias (pág. 66).

Mencionan Becker, Freeman, Hall, Cummins, \& Yuhnke (2016) la introducción de las tecnologías ofrece la posibilidad de acceder a Internet, (pág. 29). Ante lo expuesto, Rivero \& Guerrero (2017) incluyen, los dispositivos móviles han adquirido predominio en la instrucción; marcando una tendencia en los proyectos formativos posibilitan el acceso y colaboración, elementos que mejoran el rendimiento de los estudiantes (pág. 41). La evolución del Mobile -Learning busca facilitar el aprendizaje en cualquier momento y lugar, confiere la oportunidad de pensar en la educación cooperativa, y la versatilidad para promover la comunicación.

\section{Conclusiones}

- Existe un alto grado de aceptación, estimulación y perspectiva por parte de los maestros y docentes en relación al uso del celular como apoyo de refuerzo pedagógico en la enseñanza y el aprendizaje de las matemáticas, considerando que benefician en el acompañamiento pedagógico desde el proceso de sensibilización, seguimiento y monitoreo, o supervisión externa, siendo necesaria la capacitación en la utilización del Mobile Learning dentro del área de matemática.

- Desde la política institucional, se considera trascendental fortalecer la participación activa de los estudiantes mediante el uso de las tecnologías de la información y comunicación, como recurso del refuerzo académico de la Matemática; siendo así que los docentes a través de dispositivos móviles (teléfonos móviles y Smartphone) y nuevas tecnologías (teléfonos móviles, aparatos reproductores y video juegos) benefician en el desarrollo de capacidades y habilidades de los educandos para la solución de problemas y toma de decisiones.

- En referencia a la estrategia de refuerzo académico a través de la aplicación del móvil learning se fortaleció el proceso de enseñanza aprendizaje de la matemática desarrollando destrezas con criterio de desempeño para que el educando adquiera la capacidad de resolver problemas complejos con pensamiento lógico, crítico y argumentativo; elementos esenciales que favorecen en la comprensión de conceptos, el entendimiento de procesos y la práctica, desde esta perspectiva, la estrategia "reforzando nuestro conocimiento" es una herramienta que beneficia en 
a la consolidación de las competencias actitudinales, cognitivas y procedimentales.

La gratitud es la memoria del corazón, Lao - Tze

La investigación realizada va dedicada a mi querido padre que desde el cielo sigue enviando su bendición día a día, a mi abnegada madre, pilar fundamental en mi formación como profesional, a mi amada esposa y mis hijas por su paciencia, apoyo y comprensión en esta etapa de preparación y formación; a mis amigos, familiares y compañeros, al Mg. Galo López Sevilla por su de apoyo incondicional guía y orientación para culminar con el objetivo propuesto.

\section{Bibliografía}

Aguirre, G., \& Ruiz, M. (28 de Agosto de 2012). Competencias digitales y docencia: una experiencia desde la práctica universitaria. Innovación educativa, 12(59), 121141.

Angulo, M., Arteaga, E., \& Carmenates, O. (Junio de 2020). La formación de conceptos matemáticos en el proceso de enseñanza-aprendizaje de la matemática. Conrado, Cienfuegos, 16(74), 298-305. Obtenido de http://scielo.sld.cu/pdf/rc/v16n74/1990-8644-rc-16-74-298.pdf

Arcavi, A. (2 de Agosto de 2018). Hacia una visión integradora de la enseñanza y el aprendizaje de las matemáticas. Educación Matemática, 30(2), 33-48.

Basantes, A. N., Gallegos, M., \& Benítez, N. (Abril de 2017). Los dispositivos móviles en el proceso de aprendizaje de la Facultad de Educación Ciencia y Tecnología de la Universidad Técnica del Norte de Ecuador. Formación Universitaria. La Serena, 10(2), 79, 88 .

Batista, S. (2015). M-learnMat: Modelo Pedagógico para Atividades de M-learning en Matemática. Revista de Medios y Educación, 73-88.

Becker, A., Freeman, A., Hall, C., Cummins, M., \& Yuhnke, B. (2016). Reporte Horizonte del NMV/CoSN. Del Pre- Escolar al Grado 12. Austin Texa: The New Media Consortium. Revista de Educause, 1-46.

Blanco, L., Nieto, J., \& Caballero, A. (2015). Resolución de problemas de matemáticas en la formación inicial de profesores de primaria. Madrid, España: Universidad de Extremadura. 
Boaler, J. (2019). Developing Mathematical Mindsets. Developing Mathematical Mindsets: The Need to Interact with Numbers Flexibly and Conceptually, 42(4), 28,33. Obtenido de https://www.aft.org/ae/winter2018-2019/boaler

Bravino, L., \& Margaria, O. (2014). Dispositivos móviles: una experiencia en el aula de Matemática Financiera. Congreso Iberoamericano de Ciencia, Tecnología, Innovación y Educación , 84-978.

Bravo, J. (2016). Las prácticas Pedagógicas que realizan los asistentes de la educación, durante los recreos en los patios de Escuelas Públicas Básicas de Valparaíso, y su relevancia en la gestión de la convivencia. Barcelona, España: Universidad Autónoma de Barcelona.

Chávez, B., \& Chévez, M. (2019). Uso del mobile learning en el rendimiento académico. Guayaquil, Ecuador: Universidad de Guayaquil.

De la Cruz Ramírez, D., Duran, E., Romero, T., \& Álvarez, W. (19 de Diciembre de 2020). El lenguaje en el proceso de enseñanza-aprendizaje del saber matemático de la educación básica primaria, en la Institución Educativa Consuelo Araujo Noguera del Municipio de Valledupar, sector público. Universidad Popular del Cesar, 6 ( $69-74) . \quad$ Obtenido de https://revista.redipe.org/index.php/1/article/view/930/848

Delgado, C., \& Inlasaca, M. (2019). Uso de recursos tecnológicos en el proceso de enseñanza aprendizaje. Guayaquil, Ecuador: Universidad de Guayaquil, Facultad de Filosofía, Letras y Ciencias de la Educación. Obtenido de http://repositorio.ug.edu.ec/bitstream/redug/44385/1/BFILO-PD-INF21-19001.pdf

Forero, M. (2018). Implementación de una herramienta de consulta virtual: Influencia en actitudes, motivación y competencias comunicativas. Bogotá, Colombia : Pontificia Universidad Javeriana Facultad de Comunicación y Lenguaje.

Fuenmayor, G., \& Villasmil, Y. (Marzo de 2008). La percepción, la atención y la memoria como procesos cognitivos utilizados para la comprensión textua. Revista de artes y humanidades UNICA. Universidad Católica Cecilio Acosta, 9(22), 187-202.

Gallardo, I., \& Iñiguez, A. (2017). Desarrollo de un recurso didática para reforzar destrezas matemáticas en niños usando aplicativo móvil y reconocimiento de voz. Realdyc, 1-66.

Giulianelli, A., Vera, M., Rodríguez, A., Trigueros, C., González, S., \& Pons, F. (2012). Metodología para la validación de sitios web móviles. Congreos Argentino de Ciencias de la Computación, 1-36.

Guiza, M. (2011). Trabajo Colaborativo en la Web: entornos virtuales de autogestión para docentes. Palma de Mallorca, España: Universitat De Les Illes Balears. 
Hinojo, F., Aznar, I., Cáceres, M., \& Romero, J. (Diciembre de 2019). Opinión de futuros equipos docentes de educación primaria sobre la implementación del mobile learning en el aula. Revista Electrónica Educare. Scielo, 23(3), 283-299.

Instituto Nacional de Evaluación Educativa de Ecuador - Ineval. (2018). Resultados de PISA para el desarrollo. Quito, Ecuador: Ministerio de Educación.

López, E. (2018). El Mobile Learning en la Formación Continua en las Organizaciones del sector turístico. Usos y análisis de los factores para su aceptación. Barcelona, España: Universidad de Barcelona .

López, M. (2018). Trabajos de Investigación. Quito, Ecuador: Universidad Tecnológica Indoamérica.

López, M. (19 de Noviembre de 2019). Estrategias para alcanzar el aprendizaje significativo. Analítica, $15 . \quad$ Obtenido de https://monicaestrategias.blogspot.com/p/metodologia.html

Maiza, L. (2018). Desarrollo de una aplicación móvil en la enseñanza de la matemática en EGB, del Centro Escolar Ecuador. Ambato, Ecuador: Universidad Tecnológica Indoamérica.

Martínez, F. (2016). El contexto del aprendizaje móvil en América Latina. Mati-Tec. Aprendizaje móvil para el desarrollo y la inclusión, 15-34.

Mateus, J., Aran-Ramspott, S., \& Masanet, M. (2017). Análisis de la Literatura sobre Dispositivos Móviles en la Universidad Española. RIED. Revista Iberoamericana de Educación a Distacia, 1-75. Obtenido de http://revistas.uned.es/index.php/ried/article/view/17710/16047

Mojarro, Á. (2019). Mobile learning en la Educación Superior: una alternativa educativa en entornos interactivos de aprendizaje. Huelva, España: Universidad de Huelva.

Olmedo, J., \& de Alzúa, A. (2015). Desaprender para aprender . Mati-Tec. Aprendizaje móvil para el desarrollo y la inclusión , 77-109.

O'Malley. (2016). What is mobile learning. Big issues in mobile learning , 7-11.

Ortíz, J. (2021). Title: Mobile-learning as a strategy for academic reinforcement in the teaching-learning process of mathematics. 20.

Panaqué, C., \& Guerrero, C. (2017). Mobile learning y el aprendizaje de las matemáticas: el caso del proyecto MATI-TEC en el Perú. Tendencias pedagógicas. Universidad de Valencia, 30, 37-52.

Paredes, I. (2017). Estudio de las estrategias metodológicas utilizadas para la enseñanza de la Matemática en la Unidad Educativa Pedro Fermín Cevallos del cantón Cevallos. Ambato, Ecuador: Universidad Técnica de Ambato. 
Ramírez, C. (2016). Factores que inciden en el rendimiento de la matemática en los alumnos del Colegio María de la Esperanza del Municipio de Estanzuela del Departamento de Zacapa. Landívar, Guatemala: Universidad Rafael Landívar.

Reina, D., \& La Serna, N. (27 de Febrero de 2020). Revisión sistemática sobre el estado del arte de las metodologías para M-learning. Espacios, 41(6), 15.

Rivero, C., \& Guerrero, C. (2017). Mobile Learning y el Aprendizaje de las Matemáticas: El caso del proyecto Matitec. UAM. Universidad Autónoma de Madrid, 37-52.

Rodríguez, O., \& Rey, C. (2017). Los problemas sociales y su contextualización en el proceso educativo escolar: una necesidad actual. Actualidades Investigativas en Educación. Scielo, 17(2), 378-394.

Rodríguez, R., \& Vaca, V. (Septiembre de 2018). Importancia de las herramientas y entornos de aprendizaje dentro de la plataforma e-learning en las universidades del Ecuador. Edutec. Revista Electrónica De Tecnología Educativa, 65, 68-92. doi:DOI: dx.doi.org/10.21556/edutec.2018.65.1067

Ruangvanich, S., \& Nilsook, P. (Mayo de 2018). Students' Usage of Mobile Learning on Information Technology and Communication Subject for Grade 9 Students. In Proceedings of the 2018 International Conference on Distance Education and Learning . Revista Boletín Redipe, 8(10), 26-28.

Saquinga, S. (2019). Uso de la tecnología m-learning como herramienta complementaria para el aprendizaje de matemática. Ambato, Ecuador: Universidad Técnica de Ambato.

Scopeo. (26 de Enero de 2012). e-Matematicas. Obtenido de http://scopeo.usal.es/wpcontent/ uploads/2013/04/scopeom004.pdf

Sosa, C. (2021). La enseñanza e-learning de las matemáticas en Educación Primaria. Un análisis desde la perspectiva de docentes y familias. Universidad de La Laguna.

Tamayo, R., \& Álvarez, G. (2017). Análisis del diseño de cursos virtuales con Reader y Tablet en la Universidad de Holguín. Tecnología Educativa, 62-71.

Tamayo, W. (2020). Las competencias digitales de los docentes del LICGUA según el nivel de conocimiento, percepción y uso pedagógico de las TIC en los procesos de enseñanza aprendizaje. Guayaquil , Ecuador: Universidad Casa Grande.

Tapia, N. (2018). Aplicación móvil en el aprendizaje de matemáticas Básicas. Ambato: Universidad Tecnológica Indoamérica .

Trabaldo, S. (2015). Mobile Learning: Nuevas realidades en el aula. Digital Text, 397413. 
Vidal, J., Gavilondo, X., Rodríguez, A., \& Cuéllar, A. (2015). Aprendizaje Móvil. Educación Médica Superior. Scielo, 669-679.

Villanueva, S. (24 de Octubre de 2016). Alfa Cronbach, Prueba de regresión lineal, Prueba T, Chi cuadrada \& Coeficiente de correlación de Pearson. Pedagogía, 12.

Yang, J., \& Huang, R. (2016). The Learning Preferences of Digital Learners in K-12 Schools in China. EURASIA Journal of Mathematics. Science and Technology Education, 12(4), 1047-1064 (17).

Zamora, V., Soares, P., Echeverria, C., Hernández, R., \& Mijangos, C. (2015). Composite chitosan/Agarose ferrogels for potential applications in magnetic hyperethermia. Gels, 1, 69-80. 


\section{PARA CITAR EL ARTÍCULO INDEXADO.}

Ortiz Acuña, J. E., \& López Sevilla, G. (2021). Mobile-learning como estrategia de refuerzo académico en el proceso de enseñanza aprendizaje de la matemática. Explorador Digital, 5(4), 6-26. https://doi.org/10.33262/exploradordigital.v5i4.1877

\section{Ciencia \\ Ligital \\ Edtorial}

El artículo que se publica es de exclusiva responsabilidad de los autores y no necesariamente reflejan el pensamiento de la Revista Explorador Digital.

El artículo queda en propiedad de la revista y, por tanto, su publicación parcial y/o total en otro medio tiene que ser autorizado por el director de la Revista Explorador Digital.
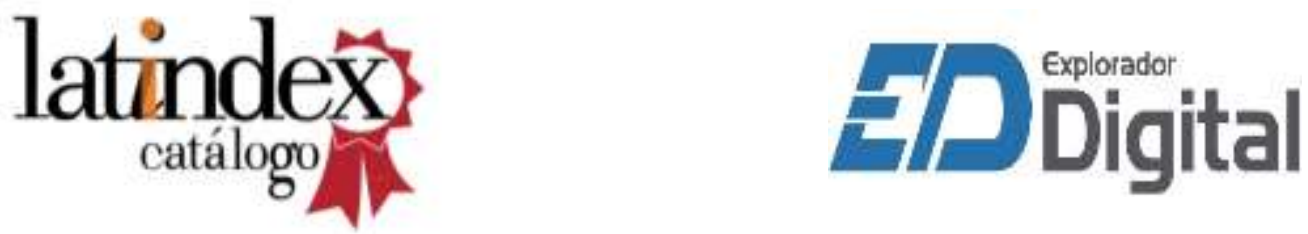